UR-1347

ER-40685-796

\title{
GELFAND-DIKII BRACKETS FOR NONSTANDARD LAX EQUATIONS
}

\author{
J.C. Brunelli \\ Ashok Das \\ Department of Physics and Astronomy \\ University of Rochester \\ Rochester, NY 14627, USA \\ and \\ Wen-Jui Huang \\ Department of Physics \\ National Tsing Hua University \\ Hsinchu, Taiwan, Republic of China
}

\begin{abstract}
$\underline{\text { Abstract }}$
We generalize the construction of Gelfand-Dikii brackets to the case of nonstandard Lax equations. We also discuss the possible origin of Kac-Moody algebras present in such systems.
\end{abstract}




\section{Introduction:}

Most integrable systems $[1,2]$ can be understood in terms of pseudo-differential operators as follows $[3,4]$. Consider, for simplicity, the formal pseudo- differential operator of the form

$$
L_{n}=\partial^{n}+u_{-1} \partial^{n-1}+u_{0} \partial^{n-2}+\ldots+u_{n-2}
$$

where ' $\partial$ ' stands for the derivative operator $\frac{\partial}{\partial x}$ and the coefficient functions, $u_{i}(x, t)$, represent dynamical variables. Then,

$$
\frac{\partial L_{n}}{\partial t_{k}}=\left[\left(L_{n}^{k / n}\right)_{+}, L_{n}\right]=-\left[\left(L_{n}^{k / n}\right)_{-}, L_{n}\right]
$$

with $k$ any positive integer which is not a multiple of $n$ defines a set of evolution equations (nonlinear) for the dynamical variables, $u_{i}(x, t)$, which are integrable. Here $\left(L_{n}^{k / n}\right)_{-}$ $\left(\left(L_{n}^{k / n}\right)_{+}\right)$denotes the part of the pseudo-differential operator $\left(L_{n}^{k / n}\right)$ containing only negative (non negative) powers of $\partial$ and the system of equations is conventionally known as the generalized KdV hierarchy. The conserved quantities (Hamiltonians) for the system can be seen to correspond to (for more details see ref. 4)

$$
\begin{aligned}
H_{k} & =\frac{n}{k} \operatorname{Tr}\left(L_{n}\right)^{k / n} \\
& \equiv \frac{n}{k} \int d x \operatorname{Res}\left(L_{n}^{k / n}\right)
\end{aligned}
$$

where Res (Residue) of a pseudo-differential operator is defined to be the coefficient of $\partial^{-1}$. These conserved charges can be easily seen from Eqs. (2) and (3) to be in involution which is necessary for the integrability of the system.

Given a pseudo-differential operator as in Eq. (1), one can define a dual operator

$$
Q_{n}=\partial^{-n} q_{-1}+\partial^{-n+1} q_{0}+\ldots+\partial^{-1} q_{n-2}
$$

which allows us to define linear functionals of the dynamical variables, $u_{i}(x, t)$, as

$$
F_{Q_{n}}\left(L_{n}\right)=\operatorname{Tr} L_{n} Q_{n}=\sum_{i=-1}^{n-2} \int d x u_{i}(x) q_{i}(x)
$$


Given this, it can be shown that the Lax equation in Eq. (2) is Hamiltonian with respect to the following Gelfand-Dikii brackets [3] (Hamiltonian structures) for appropriate Hamiltonians in Eq. (3).

$$
\begin{aligned}
\left\{F_{Q_{n}}\left(L_{n}\right), F_{V_{n}}\left(L_{n}\right)\right\}_{1} & =\operatorname{Tr} L_{n}\left[Q_{n}, V_{n}\right] \\
\left\{F_{Q_{n}}\left(L_{n}\right), F_{V_{n}}\left(L_{n}\right)\right\}_{2} & =\operatorname{Tr}\left(Q_{n} L_{n}\left(V_{n} L_{n}\right)_{+}-L_{n} Q_{n}\left(L_{n} V_{n}\right)_{+}\right) \\
& =\operatorname{Tr}\left(\left(L_{n}\left(V_{n} L_{n}\right)_{+}-\left(L_{n} V_{n}\right)_{+} L_{n}\right) Q_{n}\right)
\end{aligned}
$$

If we set $u_{-1}=0$ in Eq. (1) (which is consistent with the dynamical equations in Eq. (2)), it can be easily checked that the presence or absence of the term $\partial^{-n} q_{-1}$ in the dual makes no difference in the definitions of $F_{Q_{n}}\left(L_{n}\right)$ and the first Hamiltonian structure. However, the structure of the second Hamiltonian structure requires that $q_{-1}$ be constrained for the Lax equation to be Hamiltonian with respect to the second Hamiltonian structure [4]. Alternately, one can modify the second Hamiltonian structure in such a case to be

$$
\begin{aligned}
\left\{F_{Q_{n}}\left(L_{n}\right), F_{V_{n}}\left(L_{n}\right)\right\}_{2}^{\prime}=\operatorname{Tr} & \left(Q_{n} L_{n}\left(V_{n} L_{n}\right)_{+}-L_{n} Q_{n}\left(L_{n} V_{n}\right)_{+}\right) \\
& +\frac{1}{n} \int d x\left(\int^{x} \operatorname{Res}\left[Q_{n}, L_{n}\right]\right) \operatorname{Res}\left[V_{n}, L_{n}\right]
\end{aligned}
$$

There is a second class of integrable systems which have a nonstandard Lax representation [5]. For simplicity, let us consider the equations describing dispersive water waves (conventionally, $J_{0}=u, J_{1}=h$ and prime denotes derivative with respect to $x$ )

$$
\begin{aligned}
& \frac{\partial J_{0}}{\partial t}=\left(2 J_{1}+J_{0}^{2}-J_{0}^{\prime}\right)^{\prime} \\
& \frac{\partial J_{1}}{\partial t}=\left(2 J_{0} J_{1}+J_{1}^{\prime}\right)^{\prime}
\end{aligned}
$$

It is easy to check [5] that if we choose the pseudo-differential operator

$$
L=\partial-J_{0}+\partial^{-1} J_{1}
$$

then Eq. (8) can be written in the Lax form

$$
\frac{\partial L}{\partial t}=\left[L,\left(L^{2}\right)_{\geq 1}\right]
$$


where $\left(L^{2}\right)_{\geq 1}$ stands for the purely differential part of the pseudo-differential operator $L^{2}$ (with terms $a_{n} \partial^{n}, n \geq 1$ ). It is easy to check again that the conserved quantities of the system (which are in involution) are given by

$$
H_{n}=\operatorname{Tr} L^{n}=\int d x \operatorname{Res} L^{n} \quad n=1,2,3, \ldots
$$

The first few Hamiltonians can be directly read out from Eq. (10) to be

$$
H_{1}=\int d x J_{1} \quad H_{2}=\int d x J_{0} J_{1} \quad H_{3}=\int d x\left(J_{1}^{2}-J_{0}^{\prime} J_{1}+J_{1} J_{0}^{2}\right)
$$

The set of equations (8) can be written in the Hamiltonian form with the Poisson brackets

$$
\begin{aligned}
& \left\{J_{0}(x), J_{0}(y)\right\}_{1}=0=\left\{J_{1}(x), J_{1}(y)\right\}_{1} \\
& \left\{J_{0}(x), J_{1}(y)\right\}_{1}=\frac{\partial}{\partial x} \delta(x-y)=\partial_{x} \delta(x-y)
\end{aligned}
$$

as

$$
\begin{aligned}
& \frac{\partial J_{0}}{\partial t}=\left\{J_{0}(x), H_{3}\right\}_{1} \\
& \frac{\partial J_{1}}{\partial t}=\left\{J_{1}(x), H_{3}\right\}_{1}
\end{aligned}
$$

This defines the first Hamiltonian structure of the system. However, we note that with

$$
\begin{aligned}
& \left\{J_{0}(x), J_{0}(y)\right\}_{2}=2 \partial_{x} \delta(x-y) \\
& \left\{J_{0}(x), J_{1}(y)\right\}_{2}=J_{0}(y) \partial_{x} \delta(x-y)-\partial_{x}^{2} \delta(x-y) \\
& \left\{J_{1}(x), J_{0}(y)\right\}_{2}=J_{0}(x) \partial_{x} \delta(x-y)+\partial_{x}^{2} \delta(x-y) \\
& \left\{J_{1}(x), J_{1}(y)\right\}_{2}=\left(J_{1}(x)+J_{1}(y)\right) \partial_{x} \delta(x-y)
\end{aligned}
$$

we can also write the equations in (8) as

$$
\begin{aligned}
& \frac{\partial J_{0}}{\partial t}=\left\{J_{0}(x), H_{2}\right\}_{2} \\
& \frac{\partial J_{1}}{\partial t}=\left\{J_{1}(x), H_{2}\right\}_{2}
\end{aligned}
$$

The Hamiltonian structure in Eq. (14) defines the second structure of the theory. While these Hamiltonian structures have been obtained directly from the structure of the equations of motion as well as from other methods [6-9], a Gelfand-Dikii construction for them 
is so far lacking. In this letter, we would generalize the Gelfand-Dikii brackets to nonstandard Lax equations and bring out some connections with the Kac-Moody algebras and covariant Lax operators.

In closing this section, we note that the set of equations (8) can be mapped on to the nonlinear Schrödinger equation with the identifications

$$
\begin{aligned}
& J_{0}=-\frac{\psi^{\prime}}{\psi} \\
& J_{1}=\bar{\psi} \psi
\end{aligned}
$$

and appropriate scaling of the variables $x$ and $t$. Recently, there has been a lot of interest in this system [6-10] from various points of view. The construction of the Gelfand-Dikii brackets for such systems is, therefore, of significance.

\section{Gelfand-Dikii Brackets:}

Given the pseudo-differential operator

$$
\begin{aligned}
L & =\partial-J_{0}+\partial^{-1} J_{1} \\
& =\partial+\bar{J}_{0}+\partial^{-1} \bar{J}_{1} \quad\left(\bar{J}_{0}=-J_{0} \quad \bar{J}_{1}=J_{1}\right)
\end{aligned}
$$

let us define the dual as

$$
Q=q_{0}+q_{1} \partial^{-1}
$$

We can now define a linear functional of $\left(\bar{J}_{0}, \bar{J}_{1}\right)$ as

$$
F_{Q}(L)=\operatorname{Tr} L Q=\sum_{i=0}^{1} \int d x q_{1-i}(x) \bar{J}_{i}(x)
$$

It is now straightforward to check that

$$
\left\{F_{Q}(L), F_{V}(L)\right\}_{1}=\operatorname{Tr} L[Q, V]
$$

does, indeed, reproduce the first Hamiltonian structure in Eq. (12). However, the definitions of the brackets for the second structure in Eq. (6) or (7) fail to give the Hamiltonian structure in Eq. (14). On the other hand, it is clear that we are dealing here with a nonstandard Lax representation and consequently, the definitions of the Gelfand-Dikii brackets in Eqs. (6) and (7) may need to be modified. 
To understand the structure of the Gelfand-Dikii brackets in the present case, let us note that with

$$
\partial \omega=J_{0}=-\bar{J}_{0}
$$

we have

$$
\begin{aligned}
e^{-\omega} \partial e^{\omega} & =\partial+J_{0}=\partial-\bar{J}_{0} \equiv D \\
e^{-\omega} \partial^{n} e^{\omega} & =D^{n}=\left(\partial+J_{0}\right)^{n}=\left(\partial-\bar{J}_{0}\right)^{n}
\end{aligned}
$$

where $n$ is any integer, positive or negative. Given this, we note that

$$
\begin{aligned}
L & =\partial-J_{0}+\partial^{-1} J_{1} \\
& =e^{\omega} \hat{L} e^{-\omega}
\end{aligned}
$$

where

$$
\hat{L}=D+\bar{J}_{0}+D^{-1} \bar{J}_{1}=D-J_{0}+D^{-1} J_{1}
$$

It is now straightforward to check that

$$
\frac{\partial \hat{L}}{\partial t}=\left[\hat{L},\left((\hat{L})^{2}\right)_{+}\right]
$$

gives

$$
D^{-1} \frac{\partial J_{1}}{\partial t}-D^{-1} \frac{\partial J_{0}}{\partial t} D^{-1} J_{1}=D^{-1}\left(2 J_{0} J_{1}+J_{1}^{\prime}\right)^{\prime}-D^{-1}\left(2 J_{1}+J_{0}^{2}-J_{0}^{\prime}\right)^{\prime} D^{-1} J_{1}
$$

Comparing, we see that these give the same equations as in Eq. (8). Thus, we note that the equations (8) can be written in a standard Lax form with the pseudo-differential operator $\hat{L}$ which is expanded in the basis $D$. The Gelfand-Dikii brackets would now follow. Let us define the dual as

$$
\hat{Q}=q_{0}+q_{1} D^{-1}=e^{-\omega} Q e^{\omega}
$$

so that

$$
F_{\hat{Q}}(\hat{L})=\operatorname{Tr} \hat{L} \hat{Q}=\operatorname{Tr} L Q=F_{Q}(L)=\sum_{i=0}^{1} \int d x q_{1-i}(x) \bar{J}_{i}(x)
$$

It follows now that

$$
\left\{F_{\hat{Q}}(\hat{L}), F_{\hat{V}}(\hat{L})\right\}_{1}=\operatorname{Tr} \hat{L}[\hat{Q}, \hat{V}]
$$


would lead to the first Hamiltonian structure of the system. Using Eqs. (21), (25) and (26) it follows then that

$$
\left\{F_{Q}(L), F_{V}(L)\right\}_{1}=\operatorname{Tr} L[Q, V]
$$

would lead to the first Hamiltonian structure for the system as we have noted earlier.

The second Hamiltonian structure for a standard Lax representation is defined to be (see Eq. (6))

$$
\left\{F_{\hat{Q}}(\hat{L}), F_{\hat{V}}(\hat{L})\right\}_{2}=\operatorname{Tr}\left(\left(\hat{L}(\hat{V} \hat{L})_{+}-(\hat{L} \hat{V})_{+} \hat{L}\right) \hat{Q}\right)
$$

However, a little analysis reveals that $\hat{L}(\hat{V} \hat{L})_{+}-(\hat{L} \hat{V})_{+} \hat{L}$ is a pseudo-differential operator of leading order zero. From Eqs. (23) and (24), it then follows that this definition cannot describe the Lax equation as a Hamiltonian equation. (This is similar to the constrained case $u_{-1}=0$ we discussed earlier. See ref. 4 for details.) To remedy this, we define

$$
\begin{aligned}
& \overline{\hat{Q}}=q_{-1} D+q_{0}+q_{1} D^{-1}=q_{-1} D+\hat{Q} \\
& \overline{\hat{V}}=v_{-1} D+v_{0}+v_{1} D^{-1}=v_{-1} D+\hat{V}
\end{aligned}
$$

and note that

$$
F_{\overline{\hat{Q}}}(\hat{L})=F_{\hat{Q}}(\hat{L})=F_{Q}(L)
$$

However, we observe that $\left(\hat{L}(\overline{\hat{V}} \hat{L})_{+}-(\hat{L} \hat{V})_{+} \hat{L}\right)$ will now be an operator of leading order -1 provided

$$
v_{-1} J_{1}=v_{1}
$$

In other words, the system becomes a constrained one similar to the case of generalized KdV hierarchy which we have discussed earlier. The appropriate definition of the second Hamiltonian structure is, then,

$$
\left\{F_{\hat{Q}}(\hat{L}), F_{\hat{V}}(\hat{L})\right\}_{2}=\operatorname{Tr}\left(\left(\hat{L}(\overline{\hat{V}} \hat{L})_{+}-(\hat{L} \overline{\hat{V}})_{+} \hat{L}\right) \overline{\hat{Q}}\right)
$$

with constraints on $q_{-1}$ and $v_{-1}$ of the type in Eq. (31) and it is straightforward to check that this indeed reproduces the second Hamiltonian structure of Eq. (14). Transforming this back to the original variables we see that with

$$
\begin{aligned}
& \bar{Q}=q_{-1} \partial+Q \\
& \bar{V}=v_{-1} \partial+V
\end{aligned}
$$


where

$$
\begin{aligned}
& q_{-1} J_{1}=q_{1} \\
& v_{-1} J_{1}=v_{1}
\end{aligned}
$$

the second Hamiltonian structure can be obtained from

$$
\left\{F_{Q}(L), F_{V}(L)\right\}_{2}=\operatorname{Tr}\left(\left(L(\bar{V} L)_{+}-(L \bar{V})_{+} L\right) \bar{Q}\right)
$$

It is interesting to note that while the definition of the dual as in Eq. (29) or (33) does not change the definition of the linear functional (see Eq. (30)), it affects the structure of the first Hamiltonian structure. (This is different from the case of the generalized KdV hierarchy.) It is, therefore, more appropriate to leave the definition of the dual unchanged in the present case and modify, instead, the definition of the second bracket to be

$$
\begin{aligned}
\left\{F_{\hat{Q}}(\hat{L}), F_{\hat{V}}(\hat{L})\right\}_{2}= & \operatorname{Tr}\left(\left(\hat{L}(\hat{V} \hat{L})_{+}-(\hat{L} \hat{V})_{+} \hat{L}\right) \hat{Q}\right) \\
& +\int d x\left[\left(\int^{x} \operatorname{Res}[\hat{Q}, \hat{L}]\right) \operatorname{Res}[\hat{V}, \hat{L}]\right. \\
& \left.+\operatorname{Res}[\hat{Q}, \hat{L}] \operatorname{Res}\left(D^{-1} \hat{L} \hat{V}\right)-\operatorname{Res}[\hat{V}, \hat{L}] \operatorname{Res}\left(D^{-1} \hat{L} \hat{Q}\right)\right]
\end{aligned}
$$

In terms of the original variables, this would then give

$$
\begin{aligned}
\left\{F_{Q}(L), F_{V}(L)\right\}_{2}= & \operatorname{Tr}\left(\left(L(V L)_{+}-(L V)_{+} L\right) Q\right) \\
& +\int d x\left[\left(\int^{x} \operatorname{Res}[Q, L]\right) \operatorname{Res}[V, L]\right. \\
& \left.+\operatorname{Res}[Q, L] \operatorname{Res}\left(\partial^{-1} L V\right)-\operatorname{Res}[V, L] \operatorname{Res}\left(\partial^{-1} L Q\right)\right]
\end{aligned}
$$

These brackets are by definition antisymmetric and give the Hamiltonian structure in Eq. (14) which we know to satisfy the Jacobi identity.

\section{Kac-Moody Algebras:}

The second Hamiltonian structure of integrable systems, in general, corresponds to interesting, nontrivial symmetry algebras. Thus, for example, the Virasoro algebra arises as the second Hamiltonian structure of the $\mathrm{KdV}$ equation [11] whereas the Boussinesq equation has the $W_{3}$-algebra as its second Hamiltonian structure [12] and so on. In the present case, we note that while

$$
\left\{J_{0}(x), J_{0}(y)\right\}_{2}=2 \partial_{x} \delta(x-y)
$$


represents the $U(1)$ current algebra, the entire second Hamiltonian structure in Eq. (14) does not appear familiar. We note, however, that if we define

$$
\begin{aligned}
J(x) & =J_{0}(x) \\
T(x) & =J_{1}(x)-\frac{1}{2} J_{0}^{\prime}(x)
\end{aligned}
$$

then, the algebra in terms of these variables becomes

$$
\begin{aligned}
& \{J(x), J(y)\}_{2}=2 \partial_{x} \delta(x-y) \\
& \{T(x), J(y)\}_{2}=J(x) \partial_{x} \delta(x-y) \\
& \{T(x), T(y)\}_{2}=(T(x)+T(y)) \partial_{x} \delta(x-y)+\frac{1}{2} \partial_{x}^{3} \delta(x-y)
\end{aligned}
$$

We recognize this as the Virasoro-Kac-Moody algebra for a $U(1)$ current $[13,14]$. Since both the $\mathrm{KdV}$ and the nonlinear Schrödinger equation can be obtained from a zero curvature condition associated with $\mathrm{SL}(2, \mathbf{R})$, a hidden Virasoro algebra in the nonlinear Schrödinger equation may have been expected. It is, however, interesting to find a Virasoro-Kac-Moody algebra hidden in the nonlinear Schrödinger equation (see Eq. $\left(15^{\prime}\right)$ ).

We also note that we can define a new spin-2 generator as

$$
W(x)=T(x)-\frac{1}{4} J^{2}(x)=J_{1}(x)-\frac{1}{2} J^{\prime}(x)-\frac{1}{4} J^{2}(x)
$$

and it would satisfy the Virasoro subalgebra. However, with this redefinition, it is easy to see that the algebra (39) decouples to

$$
\begin{aligned}
\{J(x), J(y)\}_{2} & =2 \partial_{x} \delta(x-y) \\
\{W(x), J(y)\}_{2} & =0 \\
\{W(x), W(y)\}_{2} & =(W(x)+W(y)) \partial_{x} \delta(x-y)+\frac{1}{2} \partial_{x}^{3} \delta(x-y)
\end{aligned}
$$

It is interesting to note that the definition in Eq. (40) is a Miura transformation which maps the $\mathrm{KdV}$ equation to the mKdV equation [15]. The Hamiltonian structure in Eq. (41) can again be thought of as a direct sum of the Hamiltonian structures for the mKdV and the KdV systems. However, we note that while the integral of $T\left(=H_{1}\right)$ is a conserved quantity of the system, integral of $W$ is not conserved. Consequently, it is more natural to discuss the algebra in the basis of $J$ and $T$ [14]. 
Kac-Moody algebras are known to arise within the context of pseudo-differential operators when the basis of expansion is a gauge covariant derivative $[13,14]$. We do not fully understand yet the origin of the Kac-Moody algebras in the present system. However, we offer the following observations as providing a possible connection. Note that we can write

$$
\begin{aligned}
L & =\partial-J_{0}+\partial^{-1} J_{1} \\
& =\partial^{-1}\left(\partial^{2}-\partial J_{0}+J_{1}\right) \\
& =\partial^{-1}\left(\mathcal{D}^{2}+W\right)=\partial^{-1} \mathcal{L}
\end{aligned}
$$

where we have defined

$$
\begin{aligned}
\mathcal{D} & =\partial-\frac{1}{2} J_{0} \\
W & =J_{1}-\frac{1}{2} J_{0}^{\prime}-\frac{1}{4} J_{0}^{2} \\
\mathcal{L} & =\mathcal{D}^{2}+W
\end{aligned}
$$

We can think of $\mathcal{D}$ as a covariant derivative and consequently, the pseudo-differential operator $\mathcal{L}$ can be thought of as a covariant generalization of the Lax operator for the KdV system which is known $[13,14]$ to lead to a Virasoro-Kac-Moody algebra. However, more work is needed to understand the connection with the Kac-Moody algebras fully.

\section{Conclusion:}

We have proposed a generalization of the Gelfand-Dikii brackets to the case of nonstandard Lax equations. Although our discussion has been entirely within the context of the dispersive water wave equations (which can also be mapped to the nonlinear Schrödinger equation), the method is quite general and would extend readily to other nonstandard equations. We have tried to bring out the hidden Virasoro-Kac Moody algebra in the second Hamiltonian structure of the system and have offered some partial explanation for its origin. However, more work is needed for a deeper understanding of this question.

This work was supported in part by U.S. Department of Energy Grant No. DE-FG02-91ER40685. J.C.B. would like to thank CNPq, Brazil for financial support. W.-J.H. is supported by the National Science Council of the Republic of China under Grant No. NSC-83-0208-M-007-008. 


\section{$\underline{\text { References }}$}

1. L.D. Faddeev and L.A. Takhtajan, "Hamiltonian Methods in the Theory of Solitons", Springer, Berlin (1987).

2. A. Das, "Integrable Models", World Scientific (1989).

3. I.M. Gelfand and L.A. Dikii, Funct. Anal. Appl. 11, 93 (1977). M. Adler, Invent. Math. 50, 219 (1979).

L.A. Dickey, "Soliton Equations and Hamiltonian Systems", World Scientific (1991).

4. A. Das and W.J. Huang, J. Math. Phys. 33, 2487 (1992) and references therein.

5. B.A. Kupershmidt, Comm. Math. Phys. 99, 51 (1985).

6. H. Aratyn, L.A. Ferreira, J. F. Gomes and A. H. Zimerman, Nucl. Phys. B402, 85 (1993); H. Aratyn, L.A. Ferreira, J.F. Gomes and A.H. Zimerman, hep-th/9304152.

7. H. Aratyn, E. Nissimov and S. Pacheva, Phys. Lett. B314, 41 (1993).

8. L. Bonora and C. S. Xiong, Phys. Lett. B285, 191 (1992).

9. L. Bonora and C.S. Xiong, Int. J. Mod. Phys. A8, 2973 (1993).

10. M.D. Freeman and P. West, Phys. Lett. B295, 59 (1992).

11. F. Magri, J. Math. Phys. 19, 1156 (1978).

J.L. Gervais, Phys. Lett. B160, 277 (1985).

12. H.P. McKean, Adv. Math. Supp. 3, 217 (1978).

P. Mathieu, Phys. Lett. 208B, 101 (1988).

Q. Wang, P.K. Panigrahi, U. Sukhatme and Y. Keung, Nucl. Phys. B344, 196 (1990).

A. Das and S. Roy, Int. J. Mod. Phys. A6, 1429 (1991).

13. A. Das and S. Roy, J. Math. Phys. 32, 869 (1991).

14. W.J. Huang, J. Math. Phys. 35, 993 (1994).

15. R.M. Miura, J. Math. Phys. 9, 1202 (1968). 